\title{
A!
}

This is an electronic reprint of the original article.

This reprint may differ from the original in pagination and typographic detail.

Van Zanten, D. M T; Basko, D. M.; Khaymovich, I. M.; Pekola, J. P.; Courtois, H.;

Winkelmann, C. B.

\section{Single Quantum Level Electron Turnstile}

\section{Published in:}

Physical Review Letters

DOI:

10.1103/PhysRevLett.116.166801

Published: 20/04/2016

\section{Document Version}

Publisher's PDF, also known as Version of record

Please cite the original version:

Van Zanten, D. M. T., Basko, D. M., Khaymovich, I. M., Pekola, J. P., Courtois, H., \& Winkelmann, C. B. (2016). Single Quantum Level Electron Turnstile. Physical Review Letters, 116(16), 1-5. [166801].

https://doi.org/10.1103/PhysRevLett.116.166801

This material is protected by copyright and other intellectual property rights, and duplication or sale of all or part of any of the repository collections is not permitted, except that material may be duplicated by you for your research use or educational purposes in electronic or print form. You must obtain permission for any other use. Electronic or print copies may not be offered, whether for sale or otherwise to anyone who is not an authorised user. 


\title{
Single Quantum Level Electron Turnstile
}

\author{
D. M. T. van Zanten, ${ }^{1,2}$ D. M. Basko, ${ }^{1,3}$ I. M. Khaymovich, ${ }^{1,3,4}$ J. P. Pekola, ${ }^{1,2,5}$ H. Courtois, ${ }^{1,2}$ and C. B. Winkelmann ${ }^{1,2, *}$ \\ ${ }^{1}$ Université Grenoble Alpes, F-38000 Grenoble, France \\ ${ }^{2}$ CNRS, Institut Néel, F-38000 Grenoble, France \\ ${ }^{3}$ CNRS, Laboratoire de Physique et Modélisation des Milieux Condensés, F-38000 Grenoble, France \\ ${ }^{4}$ Institute for Physics of Microstructures, Russian Academy of Sciences, 603950 Nizhny Novgorod GSP-105, Russia \\ ${ }^{5}$ Low Temperature Laboratory, Department of Applied Physics, Aalto University School of Science, FI-00076 Aalto, Finland
}

(Received 11 January 2016; published 20 April 2016)

\begin{abstract}
We report on the realization of a single-electron source, where current is transported through a single-level quantum dot $(Q)$ tunnel coupled to two superconducting leads $(S)$. When driven with an ac gate voltage, the experiment demonstrates electron turnstile operation. Compared to the more conventional superconductor-normal-metal-superconductor turnstile, our superconductor-quantum-dotsuperconductor device presents a number of novel properties, including higher immunity to the unavoidable presence of nonequilibrium quasiparticles in superconducting leads. Moreover, we demonstrate its ability to deliver electrons with a very narrow energy distribution.
\end{abstract}

DOI: 10.1103/PhysRevLett.116.166801

The ability to control current flow down to the singleelectron level in nanodevices has triggered a vast activity on quantum metrological current sources in recent years [1-17]. In a quantum current source, electrons are conveyed one by one across a mesoscopic conductor, which is achieved by Coulomb repulsion. Early device geometries relied on two or more Coulomb blockaded islands in series [3]. Among the most promising recent approaches are islands with tunable barriers in 2D electron gases $[2,6,11,17]$ along with superconducting single-electron transistors [7]. Beyond metrological applications, the development of on-demand single-electron sources opens broad perspectives in the field of quantum coherent electronics and electron optics [18-22].

The superconducting single-electron transistor (SINIS) turnstile [7,23] takes advantage of the sharply defined energy gap in the density of states in superconductors, as an energy filter. A normal metallic region $(N)$ is weakly coupled to two superconducting leads $(S)$ through tunnel barriers. $N$ has to be sufficiently small to have a Coulomb charging energy $U$, which should be at least on the order of the superconducting gap in the leads, $\Delta$. Nevertheless, $N$ displays a dense set of states appearing as continuous at accessible temperatures. A finite island temperature then allows for an entire energy window $\sim k_{B} T$ of available states in $N$ for tunneling, which leads to turnstile operation errors associated to double occupation or tunneling into the wrong lead [23].

In this Letter, we demonstrate the first realization of a source of quantized dc current based on a single quantum energy level. The physical operation principle is similar to the SINIS turnstile, with the important difference that electrons are here carried by a single energy level of a quantum dot $(Q)$. After demonstrating the expected principal turnstile operation characteristics, we focus on novel electronic transport features of the superconductor-quantum-dotsuperconductor (SQS) turnstile. In particular, we show that tunneling can be tuned to occur within a narrow energy window. We theoretically compare the dominant turnstile error processes in the SQS and SINIS devices, concluding that the former has a lower sensitivity to out-of-equilibrium quasiparticles.

The fabrication of the SQS junctions described in Ref. [24] relies on the in situ creation of a nanometersized fracture in superconducting constrictions by electromigration [25], a proven technique for connecting single molecules [26] and nanoparticles [27]. Randomly dispersed gold nanoparticles of about $5 \mathrm{~nm}$ diameter can occasionally bridge the nanometric fractures, providing thereby welldefined quantum dot junctions. By using superconducting aluminum electrodes, SQS junctions can be obtained $[24,28]$. Because higher-order processes are detrimental to turnstile operation accuracy, we restrict ourselves to rather weakly coupled devices.

The relevant device parameters of the quantum dot junction are its charging energy $U$, the quantum dot orbital level spacing $\delta E$, the tunnel couplings $\gamma$, and the capacitances $C$ to the three terminals' source, drain, and gate, which we denote by indices $S, D$, and $G$, respectively. All these can be determined from transport data in static conditions, that is, measuring the current $I$ as a function of the applied bias voltage $V_{B}$ and gate voltage $V_{G}$. The $I\left(V_{B}, V_{G}\right)$ maps show typical Coulomb blockade behavior in which only a single or at most a few charge degeneracy points [Fig. 1(a)] are accessible in the available gate voltage range. We find charging energies $U>50 \mathrm{meV}$ and orbital energy level spacings $\delta E$ on the order of $1 \mathrm{meV}$ or higher. Because $\delta E \gg k_{B} T$, the thermal population beyond the 
ground state is vanishingly small, and electron transport occurs uniquely through a single orbital quantum level [29]. We focus on two devices with quite different tunnel couplings: $\mathcal{S}$ has rather symmetric tunnel couplings $\left(\gamma_{S}=2.1 \mu \mathrm{eV}, \gamma_{D}=1.4 \mu \mathrm{eV}\right)$, while $\mathcal{A}$ is strongly asymmetric $\left(\gamma_{S}=5.2 \mu \mathrm{eV}, \gamma_{D}=0.4 \mu \mathrm{eV}\right)$. Here and further, we set $\hbar=1$. The determination of all dc transport characteristics of both devices was described in detail in Ref. [24].

Superconductivity in the leads provides a hard energy filter for tunneling. The absence of quasiparticle states at energies $|E|<\Delta \approx 260 \mu \mathrm{eV}$ in the leads results in a suppression of conductance for $\left|V_{B}\right|<2 \Delta / e$ at any gate voltage, as is seen in Fig. 1(a). For turnstile operation, a small constant bias $0<\left|V_{B}\right|<2 \Delta / e$ is applied, and a periodic modulation signal with frequency $f$ and variable amplitude is added to the static gate potential. The energy difference between the $n+1$ and $n$ electron occupation numbers in the quantum dot $\epsilon(t)$ varies between $\bar{\epsilon} \pm A_{\epsilon}$, where $\bar{\epsilon}$ is controlled by the static voltages $V_{G}$ and $V_{B}$. A single electron can tunnel into the quantum dot as soon as $\epsilon(t)$ reaches the occupied states of the contact with the higher chemical potential [Fig. 1(b); right grey triangle in Fig. 1(a)]. By raising $\epsilon(t)$ via the back gate to reach the empty states above the upper gap edge in the opposite lead [left grey triangle in Fig. 1(a)], the level is emptied to that lead. By driving $\epsilon(t)$ cyclically, one electron is conveyed per cycle from the higher chemical potential lead to the other, giving rise to a dc current $I=e f$.
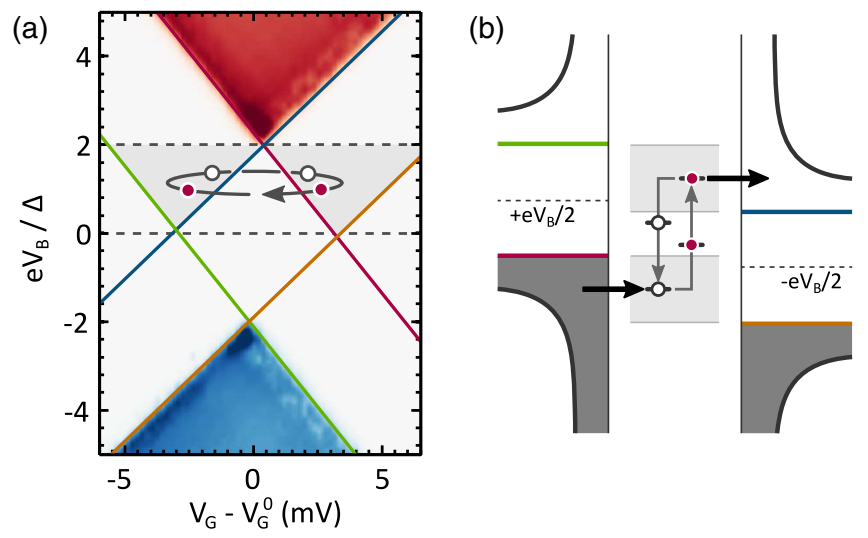

FIG. 1. (a) Experimental current map of a superconductorquantum-dot hybrid device as a function of gate and bias potential, in the absence of periodic gate drive (device $\mathcal{S}$ ). Colored solid lines correspond to the four superconducting gap edges as illustrated in (b). The device is operated as a single-level turnstile when its state is modulated periodically around its $(n, n+1)$ charge degeneracy point. The on-state currents are $I_{+}=290 \mathrm{pA}$ (red) and $I_{-}=-250 \mathrm{pA}$ (blue). (b) Energy diagram of the device with a small bias applied, illustrating electron tunneling events in and out the quantum dot. Grey areas indicate the amplitude range for solely forward tunneling, also seen in (a). Driving the turnstile with a square wave signal allows for tunneling to occur within a narrow energy window.
The combination of both of the above tunneling processes, in and out of the quantum dot, corresponds to the desired operation mode of the turnstile and will be named forward tunneling in the remainder. As shown in Fig. 1, forward tunneling requires the amplitude $A_{\epsilon}$ of the modulation of $\epsilon(t)$ to be $A_{\epsilon}>\Delta-e\left|V_{B}\right| / 2$. On the other hand, a too large modulation amplitude $A_{\epsilon}>\Delta+e\left|V_{B}\right| / 2$ will eventually allow for tunneling into or from the opposite lead. Such backtunneling processes are detrimental to current quantization, and their signature will be discussed later on.

Throughout this work, a square wave signal, with a rise time $\tau \approx 1.6 \mathrm{~ns}$ associated to the finite bandwidth of the generator, is used for modulating $\epsilon(t)$. The experimental dc current $I\left(V_{B}\right)$ measured for $\epsilon(t)$ with an amplitude $A_{\epsilon}$ around $\bar{\epsilon}=\bar{\epsilon}_{0} \equiv\left(\mu_{S}+\mu_{D}\right) / 2$ is shown in Fig. 2(a). Here $\mu_{S, D}$ are the leads' chemical potentials, with $\mu_{S}-\mu_{D}=e V_{B}$. Above the threshold voltage for forward tunneling, $V_{B}^{\mathrm{fw}}= \pm 2\left(\Delta-A_{\epsilon}\right) / e$, a broad current plateau at $I=e f$ develops. Turnstile operation is only effective for a restricted range of $\bar{\epsilon}$ [Fig. 2(b)]. The value of the turnstile current determined at the inflection point follows the predicted linear dependence on frequency [Fig. 2(c)], with a standard deviation of about $1 \%$, to which adds a systematic deficit of about $0.7 \%$ at higher frequencies. The plateaus show a small residual slope at all frequencies. This feature has instrumental origin, which is discussed in the Supplemental Material [30].

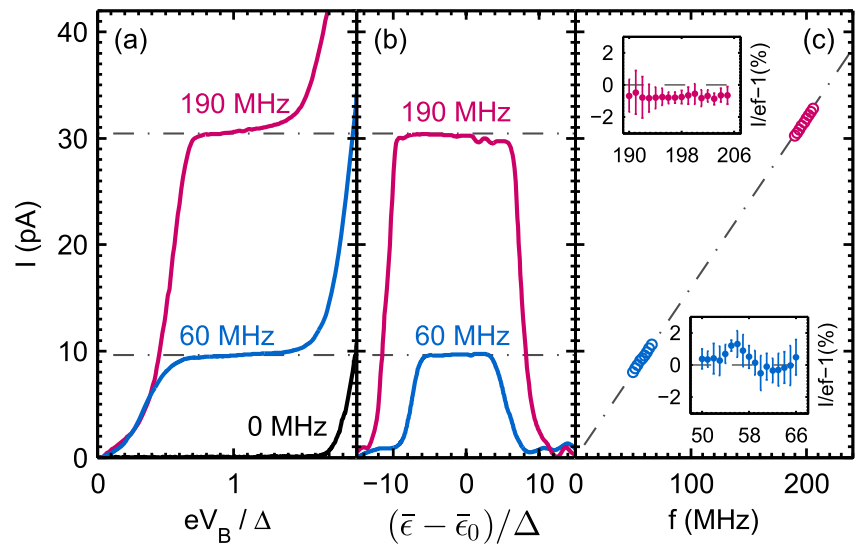

FIG. 2. (a) Current-bias traces measured near the charge degeneracy point. Characteristic plateaus appear with $I=e f$ (indicated by dashed lines) when applying a small modulation signal (magenta, $A_{\epsilon} \approx 0.64 \Delta, f=190 \mathrm{MHz}$; blue, $A_{\epsilon} \approx 1.0 \Delta$, $f=60 \mathrm{MHz}$ ) to the gate. The black trace shows the current response with no ac gate drive. (b) Current-gate traces measured for $A_{\epsilon} \approx \Delta$ and the same frequencies as in (a), at $V_{B}=\frac{3}{2} \Delta / e$ (magenta) and $V_{B}=\Delta / e$ (blue). (c) Current at the inflection point of the plateaus shown in (a) as a function of operation signal frequency. The insets highlight deviations of the normalized current $I /$ ef from 1 in both the low and high frequency ranges (all data are from device $\mathcal{S}$ ). 

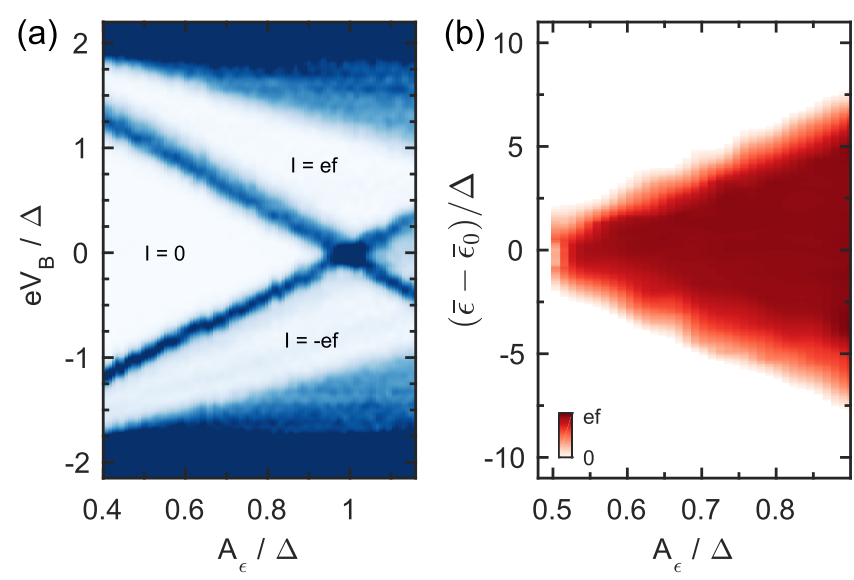

FIG. 3. (a) Color map of $\partial I / \partial V_{B}$ as a function of bias and gate modulation amplitude $\left(f=56 \mathrm{MHz}, \bar{\epsilon}=\bar{\epsilon}_{0}\right)$. Narrow blue regions corresponding to rapid increase in current separate areas of voltage-independent current (white), with values $I=0$ and $I= \pm e f$. (b) Color map of turnstile current as a function of static gate offset from degeneracy point and gate modulation amplitude $\left(f=60 \mathrm{MHz}, V_{B}=1.5 \Delta / e\right)$. All data are from device $\mathcal{A}$.

At charge degeneracy, the thresholds for the onset of both forward and backtunneling can be seen as the narrow blue stripes in Fig. 3(a). Both thresholds cross at $V_{B}=0$ when $A_{\epsilon}=\Delta$. Whereas the frequency-dependent transmission of the ac gate signal to the device is not precisely known, this crossing is used to calibrate $A_{\epsilon}$. The bright color identifies regions of voltage-independent current corresponding to $I=0$ and $I= \pm e f$, respectively.

When $\bar{\epsilon}$ is slightly detuned from $\bar{\epsilon}_{0}$ by the static gate potential, the onset of forward tunneling is linearly shifted towards larger $A_{\epsilon}$ [Fig. 1(b)]. Note that turnstile operation requires two successive tunneling events to occur. This is visible in Fig. 3(b), where the current is shown as a function of gate detuning and modulation amplitude. For larger amplitudes $A_{\epsilon}$, an increasing tolerance of the turnstile operation with respect to the proper tuning of $\bar{\epsilon}-\bar{\epsilon}_{0}$ develops.

Having evidenced electron turnstile operation, let us now identify the hallmarks of transport through a single quantum energy level. In SINIS turnstiles, backtunneling can be occasioned by electrons from the high-energy tail of the thermal energy distribution in $N$. The backtunneling probability increases, thus, steadily and smoothly as $A_{\epsilon}$ is cranked up [33]. Conversely, in a SQS turnstile, backtunneling sets in abruptly when the threshold $A_{\epsilon}=\Delta+$ $\left|V_{B}\right| / 2 e$ is exceeded. This is seen in Fig. 4(a), where at high enough modulation amplitudes, the current drops suddenly from ef. We numerically model the turnstile current dependence on $A_{\epsilon}$, both for the SINIS and the SQS turnstile, by solving the time-dependent rate equations using the measured output of the ac signal generator. In the SQS case, the instantaneous tunneling rates to each lead are found from the retarded Green's function's pole $[24,34,35]$, that is, beyond Fermi's golden rule. This is
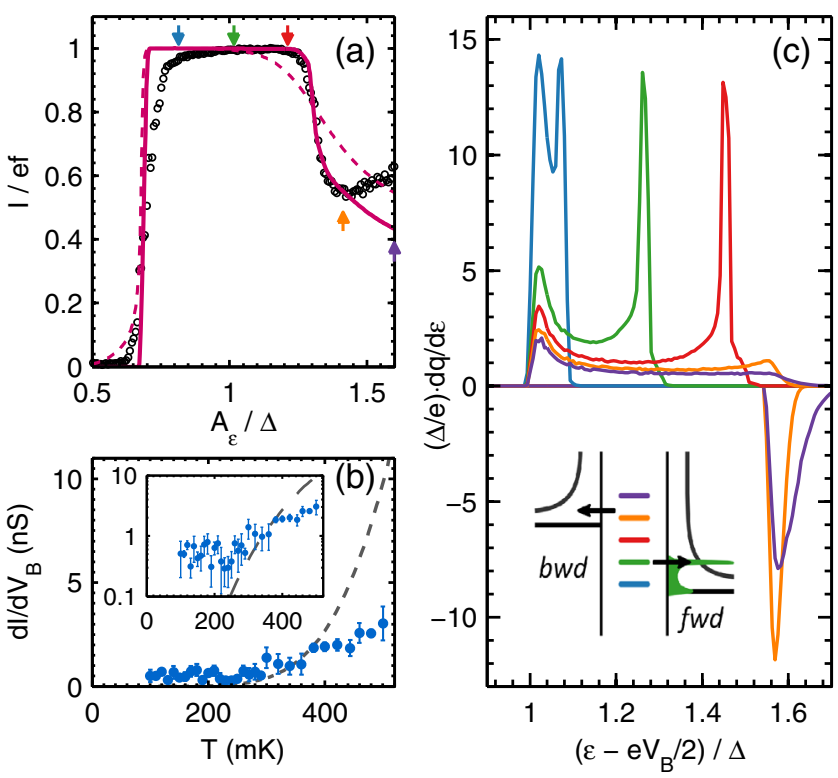

FIG. 4. (a) Turnstile current as a function of operation signal amplitude (device $\mathcal{A}, f=56 \mathrm{MHz}, \bar{\epsilon}=\bar{\epsilon}_{0}$, and $e V_{B}=0.7 \Delta$ ). The sharp decrease in current indicates the sudden onset of backtunneling. The continuous line is the numerical calculation for the SQS with all parameters determined by the device dc transport properties (see text). The dashed line is the analogous calculation for a SINIS device with normal state resistance $R_{N}=300 \mathrm{k} \Omega, U=3.0 \Delta$ and assuming quasiequilibrium of electrons in $N$ by electron-phonon relaxation [38]. The arrows indicate the values of $A_{\epsilon}$ used in (c). (b) Slope at inflection point of $I\left(V_{b}\right)$ on the turnstile plateaus, averaged over $A_{\epsilon}$, as a function of temperature (device $\mathcal{A}$ ). The dashed line is the calculation for the SINIS device, with parameters as in (a). (c) Calculation of the energy distribution of the delivered charge per cycle, for different gate drive amplitudes $A_{\epsilon}$, with parameters as in (a). The negative part of the panel displays the backtunneling contribution. The highest position of the quantum dot level, as determined by the gate modulation, is represented in the inset by the lines of corresponding colors.

particularly important near the singularities in the superconducting density of states (see the Supplemental Material [30]). The calculation [continuous line in Fig. 4(a)] nicely captures the abrupt decrease of the current as soon as the backtunneling threshold is met. For comparison, in a SINIS device with parameters taken from the most precise devices presently studied [36,37], the onset of backtunneling is markedly smoother (dashed line).

This particularly sharp onset of backtunneling is all the more pronounced if the rise time $\tau$ of $\epsilon(t)$ is short, or more precisely, if the time available for forward tunneling only is brief. If $\epsilon$ is raised to the backtunneling threshold within $\tau \ll \gamma_{S, D}^{-1}$, the probability of backtunneling may actually exceed that of forward tunneling. This means that a current inversion of magnitude up to $e f$ might eventually be produced with proper parameter combinations. This could not, however, be observed in our experiment because the 
square wave rise time is of the same order of magnitude as the inverse tunneling rate $\left(\tau \sim \gamma_{S, D}^{-1}\right)$.

To highlight the energy selectivity of the tunneling process, we calculate the energy resolved transferred charge $d q / d \epsilon$ over a half-period of an ac gate cycle using the assumptions and parameters of the calculation in Fig. 4(a). The results are shown for different values of $A_{\epsilon}$ and for both forward and backward processes in Fig. 4(c). While a certain fraction of forward tunneling occurs near the superconducting gap edge (where the lead's density of states is largest), good energy selectivity of the tunneling is achieved for sufficiently large values of $A_{\epsilon}$. For even larger $A_{\epsilon}$, backtunneling is possible, which we represent using negative values of $d q / d \epsilon$. The accuracy of the energy selectivity is ultimately limited by the tunnel coupling, but in the present experiment, it is dominated by deviations of the ac drive signal from a perfect square wave. The SQS device is expected to yield about the same energy resolution as the semiconducting ac single-electron source [18], assuming identical gate drive and tunnel couplings [39]. For comparison, the energy distribution of levitons [21] is pinned to the Fermi level.

We now move to the discussion of possible error processes of the SQS turnstile. One obvious source of error is the missed tunneling event. As the tunneling rate is finite, tunneling may be missed during the corresponding half-period, leading to $I<e f$. For a single-level quantum dot, the Fermi golden rule tunneling rate for each lead $(\alpha=S, D)$ can be written as $\Gamma_{\alpha}=(2) \gamma_{\alpha} n_{s}\left[\epsilon(t) \pm e V_{b} / 2\right]$, where $n_{s}(E)$ is the normalized quasiparticle density of states in the superconducting leads. The factor of 2 takes into account the possibility of tunneling for two spin projections and is present only for tunneling at one of the leads. For a symmetric square wave modulation of $\epsilon(t)$, the probability of missed tunneling at one of the leads can be roughly estimated as $e^{-\Gamma_{\alpha} t_{\text {eff }}}$. Here, the effective time available for tunneling $t_{\text {eff }} \approx 1 /(2 f)-\tau$ takes into account the signal rise time. At frequencies around $200 \mathrm{MHz}$, this estimate gives a current deficit of $0.8 \%$ for the device parameters of sample $\mathcal{S}$, which agrees well with the experimental value of about $0.7 \%$ [Fig. 2(c) inset]. For device $\mathcal{A}$, the missed tunneling rates at high frequencies are higher because of the tunneling bottleneck at its less transparent tunnel junction.

In turnstile operation with a normal metal island and at finite temperature, a fraction $\sim \exp \left(-\Delta / k_{B} T\right)$ of electrons has sufficiently high energy for backtunneling. In aluminum-based SINIS turnstiles, the associated error is rapidly dominant above about $300 \mathrm{mK}$ [37]. An expected hallmark of energy quantization in the turnstile operation should be a rather marked temperature insensitivity as long as $\delta E \gg k_{B} T$ and Pauli blocking of states in the leads can be neglected. We have followed the turnstile operation of device $\mathcal{A}$ as a function of temperature up to $0.5 \mathrm{~K}$, and we indeed observe the turnstile plateau to subsist through the entire temperature range, with only a rather moderate increase in error rate. We quantify the error by the $I=e f$ plateau slope $d I / d V_{B}$. As seen in Fig. 4(b), this slope shows only little dependence on temperature. For comparison, the calculation of the same for a SINIS turnstile shows a rapid divergence above about $300 \mathrm{mK}$. While thermal errors are negligible only in the low $\mathrm{mK}$ range in most reported turnstiles, the SQS device can operate up to relatively high temperatures without suffering from thermal tunneling.

An important source of errors in superconducting turnstiles is related to the presence of nonequilibrium quasiparticles in the leads, with concentration $x_{\mathrm{qp}}=n_{\mathrm{qp}} /(2 \nu \Delta)$. Here, $n_{\mathrm{qp}}$ is the quasiparticle density in the lead, and $\nu$ is the density of states (per spin projection) at the Fermi level in the normal state. Such quasiparticles can accumulate as a consequence of noise and, in particular, of the turnstile operation itself and are well known to be difficult to evacuate[36,40]. Using the diffusion model described in Ref. [36], we estimate $n_{\mathrm{qp}} \sim 10 \mu \mathrm{m}^{-3}$ near the SQS junction, yielding $x_{\mathrm{qp}} \approx 2 \times 10^{-6}$. In the SINIS turnstile, direct tunneling of such quasiparticles between one lead and the central island occurs with a rate $\sim x_{\mathrm{qp}} g \Delta$ leading to a frequency-independent leakage current. Here, $g$ is the dimensionless conductance of the tunnel junctions in units of the conductance quantum. Crucially, this leakage, which is at present the main source of errors in SINIS turnstiles [36], is suppressed for the SQS device by the lack of states in the quantum dot at the quasiparticle energy [41]. For tunneling processes of higher order in $\gamma_{S, D}$, the limitations to accuracy of the SINIS and the SQS devices are comparable. Details of the above derivations are given in the Supplemental Material [30].

To conclude, a metallic quantum dot embedded between superconducting leads allows for turnstile operation in which the charges are conveyed by a single quantum level. As a consequence, tunneling occurs within a narrow energy window determined by the level energy and broadening. Under realistic assumptions, the SQS turnstile can serve as a monochromatic on-demand single-electron source. As a next step, one can explore the possibility of spin-polarized turnstile operation by Zeeman splitting in a moderate magnetic field [30].

This work was funded by the European Union Seventh Framework Programme INFERNOS (FP7/2007-2013) under Grant No. 308850 and partially by the Russian Foundation for Basic Research (I.M. K.) and the Nanosciences Foundation (J.P. P. and I. M. K.). Samples were fabricated at the Nanofab facility at Institut Néel. We also thank F. Hekking, X. Waintal, and B. Sacépé for discussions and C. Hoarau, A. Nabet, F. Balestro, S. Samaddar, and A. de Cecco for help with the experiments. We are indebted to E. Bonet, C. Thirion, and W. Wernsdorfer for developing and sharing NanoQT. 
*clemens.winkelmann@neel.cnrs.fr

[1] L. J. Geerligs, V. F. Anderegg, P. A. M. Holweg, J. E. Mooij, H. Pothier, D. Estève, C. Urbina, and M. H. Devoret, Phys. Rev. Lett. 64, 2691 (1990).

[2] L. P. Kouwenhoven, A. T. Johnson, N. C. van der Vaart, C. J. P. M. Harmans, and C. T. Foxon, Phys. Rev. Lett. 67, 1626 (1991).

[3] H. Pothier, P. Lafarge, C. Urbina, D. Estève, and M. H. Devoret, Europhys. Lett. 17, 249 (1992).

[4] J. M. Martinis, M. Nahum, and H. D. Jensen, Phys. Rev. Lett. 72, 904 (1994).

[5] Y. Ono and Y. Takahashi, Appl. Phys. Lett. 82, 1221 (2003).

[6] M. D. Blumenthal, B. Kaestner, L. Li, S. Giblin, T. J. B. M. Janssen, M. Pepper, D. Anderson, G. Jones, and D. A. Ritchie, Nat. Phys. 3, 343 (2007).

[7] J. P. Pekola, J. J. Vartiainen, M. Möttönen, O.-P. Saira, M. Meschke, and D. V. Averin, Nat. Phys. 4, 120 (2008).

[8] B. Kaestner, V. Kashcheyevs, S. Amakawa, M. D. Blumenthal, L. Li, T. J. B. M. Janssen, G. Hein, K. Pierz, T. Weimann, U. Siegner, and H. W. Schumacher, Phys. Rev. B 77, 153301 (2008).

[9] V. Siegle, C.-W. Liang, B. Kaestner, H. W. Schumacher, F. Jessen, D. Koelle, R. Kleiner, and S. Roth, Nano Lett. 10, 3841 (2010).

[10] F. Giazotto, P. Spathis, S. Roddaro, S. Biswas, F. Taddei, M. Governale, and L. Sorba, Nat. Phys. 7, 857 (2011).

[11] S. P. Giblin, M. Kataoka, J. D. Fletcher, P. See, T. J. B. M. Janssen, J. P. Griffiths, G. A. C. Jones, I. Farrer, and D. A. Ritchie, Nat. Commun. 3, 930 (2012).

[12] B. Roche, R.-P. Riwar, B. Voisin, E. Dupont-Ferrier, R. Wacquez, M. Vinet, M. Sanquer, J. Splettstoesser, and X. Jehl, Nat. Commun. 4, 1581 (2013).

[13] M. R. Connolly, K. L. Chiu, S. P. Giblin, M. Kataoka, J. D. Fletcher, C. Chua, J. P. Griffiths, G. A. C. Jones, V. I. Fal'ko, C. G. Smith, and T. J. B. M. Janssen, Nat. Nanotechnol. 8, 417 (2013).

[14] J. P. Pekola, O.-P. Saira, V. F. Maisi, A. Kemppinen, M. Möttönen, Y. A. Pashkin, and D. V. Averin, Rev. Mod. Phys. 85, 1421 (2013).

[15] G. Yamahata, K. Nishiguchi, and A. Fujiwara, Nat. Commun. 5, 5038 (2014).

[16] A. Rossi, T. Tanttu, K. Y. Tan, I. Iisakka, R. Zhao, K. W. Chan, G. C. Tettamanzi, S. Rogge, A. S. Dzurak, and M. Möttönen, Nano Lett. 14, 3405 (2014).

[17] F. Stein, D. Drung, L. Fricke, H. Scherer, F. Hohls, C. Leicht, M. Götz, C. Krause, R. Behr, E. Pesel, K. Pierz, U. Siegner, F. J. Ahlers, and H. W. Schumacher, Appl. Phys. Lett. 107, 103501 (2015).

[18] G. Fève, A. Mahe, J.-M. Berroir, T. Kontos, B. Plaçais, D. Glattli, A. Cavanna, B. Etienne, and Y. Jin, Science 316, 1169 (2007).
[19] R. McNeil, M. Kataoka, C. Ford, C. Barnes, D. Anderson, G. Jones, I. Farrer, and D. Ritchie, Nature (London) 477, 439 (2011).

[20] S. Hermelin, S. Takada, M. Yamamoto, S. Tarucha, A. D. Wieck, L. Saminadayar, C. Bäuerle, and T. Meunier, Nature (London) 477, 435 (2011).

[21] J. Dubois, T. Jullien, F. Portier, P. Roche, A. Cavanna, Y. Jin, W. Wegscheider, P. Roulleau, and D. C. Glattli, Nature (London) 502, 659 (2013).

[22] V. Freulon, A. Marguerite, J.-M. Berroir, B. Plaçais, A. Cavanna, Y. Jin, and G. Fève, Nat. Commun. 6, 6854 (2015).

[23] D. V. Averin and J. P. Pekola, Phys. Rev. Lett. 101, 066801 (2008).

[24] D. M. T. van Zanten, F. Balestro, H. Courtois, and C. B. Winkelmann, Phys. Rev. B 92, 184501 (2015).

[25] H. Park, A. K. Lim, A. P. Alivisatos, J. Park, and P. L. McEuen, Appl. Phys. Lett. 75, 301 (1999).

[26] H. Park and P. L. McEuen, Nature (London) 407, 57 (2000).

[27] F. Kuemmeth, K. I. Bolotin, S.-F. Shi, and D. C. Ralph, Nano Lett. 8, 4506 (2008).

[28] C. B. Winkelmann, N. Roch, W. Wernsdorfer, V. Bouchiat, and F. Balestro, Nat. Phys. 5, 876 (2009).

[29] D. C. Ralph, C. T. Black, and M. Tinkham, Phys. Rev. Lett. 74, 3241 (1995).

[30] See the Supplemental Material http://link.aps.org/ supplemental/10.1103/PhysRevLett.116.166801, which includes Refs. [31,32], for a discussion of capacitive crosstalk effects and theoretical aspects of the Green's functions calculations of both the tunneling rates and error processes.

[31] V. F. Maisi, Ph. D. thesis, Aalto University, 2009.

[32] I. L. Aleiner and L. I. Glazman, Phys. Rev. Lett. 77, 2057 (1996).

[33] A. Kemppinen, S. Kafanov, Y. A. Pashkin, J. S. Tsai, D. V. Averin, and J. P. Pekola, Appl. Phys. Lett. 94, 172108 (2009).

[34] A. Levy Yeyati, J. C. Cuevas, A. López-Dávalos, and A. Martín-Rodero, Phys. Rev. B 55, R6137 (1997).

[35] K. Kang, Phys. Rev. B 57, 11891 (1998).

[36] H. S. Knowles, V. F. Maisi, and J. P. Pekola, Appl. Phys. Lett. 100, 262601 (2012).

[37] S. Nakamura, Y. Pashkin, J.-S. Tsai, and N.-H. Kaneko, Physica C (Amsterdam) 504, 93 (2014).

[38] F. Giazotto, T. Heikkilä, A. Luukanen, A. Savin, and J. Pekola, Rev. Mod. Phys. 78, 217 (2006).

[39] This is because the integral over the density of states of a superconductor is the same as in the normal state.

[40] O.-P. Saira, A. Kemppinen, V. F. Maisi, and J. P. Pekola, Phys. Rev. B 85, 012504 (2012).

[41] Another contribution of the nonequilibrium quasiparticles, the Pauli blocking term, may also be significantly suppressed in the SQS turnstile depending on the quasiparticle distribution function (see the Supplemental Material [30] for details). 